with unrelated external controls. The co-twin control method compared twins with dementia to their unaffected twin sibling, thus testing the importance of depression as a risk factor while controlling for genetic background and early shared environmental experiences. Results: Case-control results showed that individuals with a history of depression were 1.72 times more likely to develop dementia than those without a history of depression $(\mathrm{CI}=1.07,2.76, \mathrm{p}=0.0212)$. Risk was greater for depression when the first episode was closer to dementia onset; for each one-year increase in the difference between age of onset of depression and age of onset of dementia or censored age, the risk of developing dementia decreased by $8.1 \%$ ( $\mathrm{p}=$ 0.0031). Co-twin control analysis found that individuals with a history of depression were 3.19 times more likely to develop dementia $(\mathrm{CI}=1.35,7.55, \mathrm{p}=$ 0.0082). Conclusions: These findings suggest that depression is a prodrome, rather than a risk factor for dementia.

Research funded by NIA grants R01 AG08724 and a Zenith Award from the Alzheimer's Association.

\section{P-155 THE ESTABLISHMENT OF AUSTRALIAN DEMENTIA COLLABORATIVE RESEARCH CENTRES: A NEW MODEL}

Marc Budge on behalf of DCRC2. The Australian National University, Garran, ACT, Australia. Contact e-mail: marc.budge@anu.edu.au

The 2005 Australian Government Budget provided \$AUD 320.6 million over five years for the Dementia Initiative (www.health.gov.au/internet/wcms/publishing.nsf/Content/2005dementiabudgetinitiative-1-lp). As part of Program One, Dementia - A National Health Priority, three integrated Dementia Collaborative Research Centres (DCRCs), each with a specific focus, have been established. The goal of the DCRCs are to facilitate, undertake and promote research, assist in the translation of research outcomes into clinical practice and develop guidelines and tools for use by health professionals at individual and population health program levels with respect to dementia. The DCRC number 2 (DCRC2) led by the Australian National University (ANU) will coordinate, undertake, facilitate and promote reviews and research within the area of Prevention, Early Intervention and Risk Reduction in Dementia. The initial centre members include the Australian National University alongside Alzheimer's Australia Victoria (AAV), Edith Cowan University (ECU), University of Canberra (UC), University of Melbourne (UMEL) and the University of Queensland (UQ). During 2007-2009, DCRC2 members will undertake 25 projects within 4 program areas: A. Understanding Risks for Dementia: This program will include identification and review of "established" and new or emerging risk factors; genetic-biological markers, environment and lifestyle factors and their interaction in developing dementia will also be examined. B. Development of Diagnostic Tools and Algorithms for Early Dementia: Diagnostic tools and pathways for cognitive testing (e.g. checklists, computerized and on-line or self-administered tools) will be reviewed and developed. Neuro-imaging projects will include the development of the Australian Normative and Dementia Imaging Database (ANDI) whilst methodological development of dementia-related biomarkers will also be undertaken. C. Development of Risk Reduction Interventions: Projects under this program are aimed at translating the knowledge derived from programs A and B into health professional and public awareness, knowledge and action. D. Modelling Impacts of Risk Reduction on Cognitive Decline and Dementia: Projects will develop methodology for, and model, the impact of dementia risk reduction programs. The launch of the Australian Government's Dementia Initiative and this new model of collaborative DCRCs will be described. In so doing, it is hoped that opportunities for collaborative research between Australian and international researchers may be further fostered.

\section{P-156}

\section{LOW SERUM BIOAVAILABLE TESTOSTERONE LEVELS ARE ASSOCIATED WITH MILD COGNITIVE IMPAIRMENT IN CHINESE OLDER MEN}

Leung-Wing Chu ${ }^{1,2}$, Sidney Tam ${ }^{2}$, Karen S.L. Lam ${ }^{1},{ }^{1}$ The University of Hong Kong, Hong Kong, Hong Kong; ${ }^{2}$ Queen Mary Hospital, Hong Kong, Hong Kong. Contact e-mail: lwchu@hkucc.hku.hk
Background: There is no previous report on the relationship of serum testosterone levels, mild cognitive impairment and Alzheimer's disease in the Chinese elderly. Objective(s): The objective of the present study was to investigate the associations of serum total and bioavailable testosterone levels with mild cognitive impairment and Alzheimer's disease Methods: A cross-sectional study of 100 Chinese older men aged $\geq 60$ who were assessed by a detailed clinical, laboratory and neuropsychological assessment as well as fasting morning blood serum total and bioavailable testosterone levels. We established the diagnosis of dementia by the DSM-IV criteria, Alzheimer's disease (AD) by the NINCDS-ADRDA criteria and mild cognitive impairment (MCI) by the Petersen's criteria. Results: 52, 30 and 18 of the subjects had normal cognitive function (N), MCI and AD, respectively. The mean serum bioavailable (but not the total testosterone) levels showed a statistically significant difference between the N, MCI and $\mathrm{AD}$ groups $\{$ mean $(\mathrm{sem})$ serum bioavailable levels were $1.49(0.13)$, $0.95(0.11)$ and $0.91(0.12) \mathrm{nmol} / \mathrm{L}$, respectively, for $\mathrm{N}$, MCI and AD groups, $p=0.003$, 1-way ANOVA $\}$. Significant differences were present between the $\mathrm{N}$ and $\mathrm{MCI}$ as well as the $\mathrm{N}$ and AD subjects in the subgroup analysis (post-hoc, Bonferroni). Conclusions: Low serum bioavailable testosterone levels were significantly associated with mild cognitive impairment as well as Alzheimer's disease in Chinese older men.

\section{P-157 FACTORS ASSOCIATED WITH THE IMPACT OF SUSCEPTIBILITY GENETIC TESTING OF ALZHEIMER'S DISEASE}

Winston W. Chung ${ }^{1}$, Clara A. Chen ${ }^{1}$, Susan Hiraki ${ }^{1}$, Anil K. Nair ${ }^{1}$, Jeffrey Scott Roberts ${ }^{2}$, Robert C. Green ${ }^{1},{ }^{1}$ Boston University School of Medicine, Boston, MA, USA; ${ }^{2}$ University of Michigan School of Public Health, Ann Arbor, MI, USA. Contact e-mail: wchung47@bu.edu

Background: The REVEAL Study is a randomized clinical trial (RCT) that examines the impact of providing genetic risk assessment, including APOE genotype disclosure, to unaffected first-degree relatives of AD patients. Although subjects reported positive experiences during the course of the study, factors underlying variations in psychological impact are not clearly understood. Objective: To identify demographical, attitudinal, or psychological factors that are associated with variability in response to susceptibility genetic testing for AD. Methods: A total of 280 participants received risk assessment, including APOE genotype disclosure, in a multistage education and counseling protocol. A modified version of the Multidimensional Impact of Cancer Risk Assessment (MICRA) measure, adapted for $\mathrm{AD}$, was used in this analysis to ascertain the impact of genetic testing at 6 week and 12 month visit. The total score is summed from three subscales: distress, uncertainty, and positive experience. Unlike many other commonly used measures in the genetic testing literature (e.g., general scales of anxiety and depression), the MICRA is designed to assess responses specific to genetic risk assessment. The following variables were examined as predictors of MICRA score: age, gender, race, genotype (e4 status), whether or not subjects told others about their genotype, CES-D baseline score (depressive symptom measure), and BAI baseline score (anxiety symptom measure). Results: Multivariate analysis showed that age, gender, race, and BAI baseline score were not associated with MICRA scores. However, when adjusted for these variables, e4 status $(\mathrm{p}=.0001$ at 6 weeks; $\mathrm{p}=.0001$ at 12 month), telling others about one's genotype ( $\mathrm{p}=0.03$ at 6 weeks; $\mathrm{p}=0.01$ at 12 month) and CES-D baseline score $(\mathrm{p}=0.04$ at both time points) were significant predictors of MICRA score. Conclusion: Within the context of overall positive outcomes after disclosure, APOE status is a strong predictor of less favorable psychological response to genetic risk information. Those subjects who had higher baseline scores on a measure of depressive symptoms and/or did not tell others about their genotype results also experienced less favorable responses. Results suggest that participants' APOE status, social support, and baseline levels of depression may warrant particular attention in future predictive testing and counseling scenarios. 
Supported by NIH Grants HG/AG02213 (REVEAL Study), AG13846 (Boston University Alzheimer's Disease Core Center), and M01-RR00533 (Boston University GCRC). Boston University School of Medicine Summer Research Scholar Grant.

\section{P-158}

\section{ELEVATED GLUCOCORTICOIDS AMONG VICTIMS OF INTIMATE PARTNER VIOLENCE AS A BIOMARKER FOR ALZHEIMER'S DISEASE}

James M. DeCarli, University of Southern California, Los Angeles, CA, USA. Contact e-mail: jdecarli@ladhs.org

Background: Chronic stress has been shown to be a contributing factor to structural changes in the hippocampus and amagdala, and while structural changes of the hippocampus have been associated with an increased risk of Alzheimer's Disease, no studies have implicated an association between neuroplasticity and atrophy of these brain regions due to emotional and psychological abuse that occurs in intimate partner violence. Objective(s): 1) Determine how chronic stress contributes to structural changes within the hippocampus; 2) Describe the physiological and neurological responses due to exposure to stress hormones; 3) Are structural changes of the hippocampus associated with an increased risk of Alzheimer's disease; 4) Describe how behavioral and psychophysiological outcomes of intimate partner violence contributes to neuroplasticity; and 5) Provide a systematic review of literature on neural imaging techniques to confirm if glucocorticoids are associated with hippocampal atrophy, and provide evidence that hippocampal atrophy is associated with AD. Methods: Several systematic reviews were conducted. First, on neural imaging techniques on hippocampal atrophy in Alzheimer's Disease, secondly, on the acute and chronic affects of glucocorticoids on memory, and thirdly the neurobehavioral characteristics of victims of emotional and psychological abuse and those diagnosed with Alzheimer's Disease. Results: A descriptive analysis from neurobehavioral, physiology, and intimate partner violence literature identified how emotional and psychological abuse among intimate partner violence results in depression, and while neural imaging studies have shown that depression is associated with hippocampal atrophy and hippocampal atrophy is a hallmark of Alzheimer's Disease. Conclusions: The results of this review suggests by using neural imaging techniques; Positron Emission Tomography can be used to help rule-out Alzheimer's Disease by hippocampal volume measurement to help reduce misdiagnosis of Alzheimer's Disease in patients 65 years of age and older and further provides a foundation to support the need to design cognitive behavioral interventions to reduce emotional and psychological abuse. Such interventions can be evaluated and outcomes analyzed to measure efficacy that will ultimately provide a baseline to view Alzheimer's Disease causality from a multidisciplinary approach and expanding further psychoneuroimmunology research.

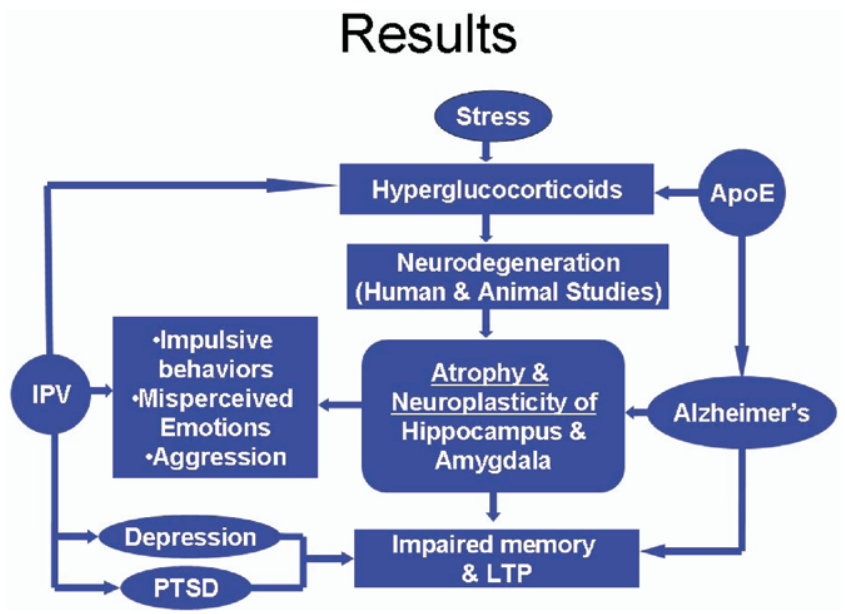

\section{VITAMIN B12, APOLIPOPROTEIN E GENOTYPE,} AND COGNITIVE PERFORMANCE

Lei Feng ${ }^{1}$, Tze-Pin $\mathrm{NG}^{1}$, Mathew NITI ${ }^{1}$, Keng-Bee $\mathrm{YAP}^{2}$, Ee-Heok KUA ${ }^{1},{ }^{\prime}$ National University of Singapore, Singapore, Singapore; ${ }^{2}$ Alexandra Hospital, Singapore, Singapore. Contact e-mail: fenglei@nus.edu.sg

Background: The relationship between vitamin B-12 and cognitive function in older people is unclear because studies have reported inconsistent results. The role of Apolipoprotein E (APOE) e4 as a genetic predisposition factor in the relationship between B-12 and cognitive function is not well studied. Objective(s): To examine the interaction between Vitamin B-12 and APOE genotype on cognitive performance in high functioning older Chinese adults. Methods: Serum vitamin B-12 and APOE genotyping were determined in 493 Chinese aged $\geq 55$ years with Mini Mental State Examination (MMSE) score $\geq 24$ and fully Independent on daily living activities. Cognitive performance was assessed for attention and working memory (Digit Span and Spatial Span), verbal learning and memory (Rey Auditory Verbal Learning Test), visual memory (Visual Reproduction), language (Categorical Verbal Fluency), executive function (Design Fluency), information processing speed (Symbol Digit Modality Test). Multiple linear regressions with controlling for potential confounding were performed for APOE e4 allele carrier and non-e4 allele carriers, with significance testing of B12 (modeled as both continuous and dichotomous variables) and its interaction term with APOE. Results: Vitamin B-12 was not associated with any cognitive measures in Non-APOE e4 carriers in the sample overall or in non-APOE e4 carriers. In APOE e4 carriers, log transformed vitamin B-12 was positively and significantly associated with RAVLT immediate recall $($ Beta $=0.281, \mathrm{P}=0.028), \mathrm{VR}$ immediate recall $(B e t a=0.214, P=0.044)$, SDMT written (Beta $=0.271, P=0.049)$. The interaction between low vitamin B-12 and APOE e4 allele was significant for MMSE (Beta $=-0.101, \mathrm{P}=0.027$ ), RAVLT immediate recall (Beta=$0.117, \mathrm{P}=0.027$ ), SDMT written (Beta $=-0.114, \mathrm{P}=0.029$ ) and SDMT oral (Beta $=-0.111, \mathrm{P}=0.049)$. Conclusions: In this group of community-dwelling Chinese older adults, the association between vitamin B-12 and cognitive performance was modified by APOE genotype. Our findings suggest that genetic disposition may make individuals more vulnerable to the influence of specific micronutrient. Further longitudinal study is warranted to examine whether there is interaction of vitamin B-12 and APOE genotyping on cognitive decline and incidence of dementia.

\section{P-160 MOTIVATIONAL RESERVE: A NEW CONCEPT COMPLEMENTING COGNITIVE RESERVE}

Simon Forstmeier, Andreas Maercker, University of Zurich, Zurich, Switzerland.Contact e-mail: s.forstmeier@psychologie.unizh.ch

Background: In Alzheimer's disease, several categories of psychological resources have shown to influence the incidence and progression of this disorder. The concept of "Cognitive Reserve" (CR) was introduced by Stern and others to explain the less than perfect relationship between the degree of brain pathology and its clinical manifestation. We will present a model based on the work of Bandura, Karoly, Pintrich and others that integrates cognitive and motivational competencies in the prediction of academic achievement and health effects (i.e., dementia). The concept of "Motivational Reserve" (MR) will be introduced to account for the observation that motivational resources such as self-efficacy, optimism, motivation regulation, and emotion regulation are associated with a slowing down of cognitive decline and later clinical manifestation of dementia. Objective(s): The aim of the series of studies is to evaluate newly developed instruments of premorbid motivational competence. So far, established instruments of motivational resources rely mainly on selfreport and refer to the present state. New instruments will be described that allow the estimation of premorbid motivational competence using different ways than self-report. Methods: Two studies were conducted with elderly ( $\geq 65$ years) non-demented subjects. The first study ( $N=106,51 \%$ female, mean age 73 years) used established and new questionnaire measures of motivational self-concept (e.g., self-efficacy) and motivational self-regulation 